\title{
Karrieremöglichkeiten am Schnittpunkt zwischen Medizin und Forschung
}

Atanas Todorov, Shuyang Traub,

Im Namen des Vorstands der Schweizerischen MD-PhD Gesellschaft Laurence Feldmeyer

Die American Physician Scientist Association (APSA) wurde 2004 von MD-PhD-Studenten und forschenden Ärzten als Austauschplattform gegründet [1]. Während der Gründungsphase gab es viel Einfluss aus Europa, wo zur selben Zeit nationale und internationale MD-PhD-Treffen stattfanden. Die damals noch sehr junge Swiss MD-PhD Association (SMPA) übte eine Vorbildrolle aus [2].

APSA ist eine nationale Vereinigung geworden, die erfolgreich die Interessen forschender Ärzte an Akademien und in der Klinik vertritt. Dank ihrer Bemühungen sind zahlreiche MD-PhD-Programme und kombinierte Stellen für Forschung und Klinik entstanden.

Als wichtige Grundlage ihrer Arbeit dient eine jährliche Umfrage unter Mitgliedern und Medizinstudenten. Dadurch entsteht eine umfangreiche Datenbank zur aktuellen Karrieresituation und den

\section{Vereinigungen wie APSA und SMPA können erfolgreich die Interessen forschender Ärzte an Akademien und in der Klinik vertreten.}

Korrespondenz: Atanas Todorov Hebelstrasse 20 CH-4031 Basel
Bedürfnissen von forschenden Ärzten [3-5] (vgl. Kasten). Eine ähnliche Umfrage wurde dieses Jahr zum ersten Mal von der SMPA in Zusammenarbeit mit APSA unter Schweizer MD-PhD-Studenten und -Absolventen durchgeführt.

\section{Networking für forschende Ärzte über Hierarchiestufen hinweg}

Das jährliche nationale Treffen ist das Herzstück der APSA. Der Kerngedanke ist die Nutzung, Erhaltung und Erneuerung eines grossen Netzwerks an forschenden Ärzten. Während drei Tagen werden wissenschaftliche Referate und Networking für jeden Karrieregrad angeboten. Studenten können sich über diverse Universitätsprogramme informieren und erfahrene Mentoren befragen. Amerikanische MD-PhD-Kandidaten müssen die Vorklinik und das erste amerikanische Staatsexamen bestehen, um sich für ein drei- bis fünfjähriges $\mathrm{PhD}$-Programm anzumelden. Dementsprechend ist Forschung für sie ein Teil ihres Studiums. Nach dem PhD werden zwei klinische Jahre und der zweite Teil des Staatsexamens absolviert. Im Vergleich dazu schliessen MD-PhD-

\section{Les possibilités de carrière entre médecine et recherche}

Depuis 2004, l'Association américaine des médecins-chercheurs (APSA) organise chaque année une rencontre entre médecins et étudiants intéressés par la recherche et les programmes MD-PhD, qui met l'accent sur le réseautage et les perspectives professionnelles. Les activités incluent un petit-déjeuner de mentorat, lors duquel Jaimo Ahn, professeur assistant d'orthopédie à l'Université de Pennsylvanie, a présenté son point de vue sur les opportunités offertes par la recherche en chirurgie. Les participants ont également eu la possibilité de visiter des laboratoires et des cliniques, dont celle de l'Université de I'Illinois à Chicago, où travaille Jennifer Kwan, interne et post-doctorante, et de rencontrer José Oberholzer, directeur du centre de transplantation d'îlots de Langerhans à Genève jusqu'en 2002 et directeur de la chirurgie de la transplantation à Chicago depuis 2007. Même si les Etats-Unis restent le pays où tout semble possible, la Suisse n'a pas à craindre la comparaison.

Studenten in der Schweiz ihr Medizinstudium ab und beginnen danach das zusätzliche PhD-Studium. Forschende Ärzte können während des Treffens ebenfalls unter ihresgleichen über Erfolge, Misserfolge und andere Erfahrungen reden. Es findet gleichzeitig das Treffen der Association of American Physicians und der American Society for Clinical Investigation statt, was die Sponsorsuche und die Organisation für die APSA erleichtern.

Zur Karriereplanung sind zahlreiche Informationen vorhanden und es wird aktiv für die klinische Weiterbildung nach dem PhD geworben (Tab. 1). Der persönliche Kontakt mit erfahrenen Fakultätsmitgliedern steht an erster Stelle. Gerade hier entsteht der Eindruck, dass viel Wert auf den Nachwuchs gelegt wird. 
Tabelle 1

Beispiel eines kombinierten Assistenzarzt/Postdoc-Programms, wie es an der University of lowa, Carver College of Medicine, angeboten wird (Quelle: Info-Broschüre der University of lowa). Zum Vergleich in der letzten Spalte die Weiterbildung in allgemeiner Innerer Medizin in der Schweiz (Quelle: www.fmh.ch/files/pdf14/aim_wbp_d.pdf).

\begin{tabular}{|c|c|c|c|}
\hline & $\begin{array}{l}\text { Innere Medizin } \\
\text { (USA) }\end{array}$ & $\begin{array}{l}\text { Pathologie } \\
\text { (USA) }\end{array}$ & $\begin{array}{l}\text { Innere Medizin } \\
\text { (FMH Schweiz) }\end{array}$ \\
\hline Allgemeine Assistenzzeit & 2 Jahre & 3-4 Jahre & 3 Jahre \\
\hline $\begin{array}{l}\text { Ausbildung in klinischer } \\
\text { Subspezialität }\end{array}$ & 1-2 Jahre & $1 \mathrm{Jahr}$ & 2 Jahre \\
\hline $\begin{array}{l}\text { Wissenschaftliche Ausbildung } \\
\text { (Postdoc) }\end{array}$ & 3 Jahre & 1-2 Jahre & Max. $1 / 2$ Jahr \\
\hline $\begin{array}{l}\text { Arbeit in Poliklinik während } \\
\text { wissenschaftlicher Ausbildung }\end{array}$ & $1 / 2$ Tag pro Woche & - & - \\
\hline Gesamte Ausbildungszeit & 6-7 Jahre & 5-7 Jahre & 5-6 Jahre \\
\hline
\end{tabular}

Wie jedes Jahr fand auch das diesjährige 10. Treffen im Fairmont Hotel mitten in der Chicagoer Downtown statt. Unter den Anwesenden waren Nobelpreisträger wie Peter Agre, Robert Horvitz, Joseph Goldstein und Brian Kobilka. Ein Höhepunkt

\section{Eine Herausforderung: die Verbindung von medizinischem Alltag und Forschung.}

war die Ansprache von Anthony Fauci, Direktor des National Institute of Allergy and Infectious Diseases (NIAID), über die Entdeckung von HIV und die Zusammenarbeit mit fünf amerikanischen Präsidenten. Forschende Ärzte berichteten über ihre Erfahrungen, wie zum Beispiel Jaimo Ahn, Assistenzprofessor für Orthopädie am Universitätsspital von Pennsylvania. Er schilderte seinen Werdegang und die Perspektiven für Chirurgen in der Forschung.

\footnotetext{
Nützliche Webseiten

www.physicianscientists.org

Webseite der American Physician Scientist Association (APSA). Die Mitgliedschaft kostet jährlich 25 Dollar und bietet vergünstigte Anmeldungen zu einigen Kongressen, sowie Informationen zu Programmen, Seminare übers Internet (sog. Webinars) und Foren, in denen persönliche Erfahrungen ausgetauscht werden. Ebenfalls nützlich: Man kann andere Mitglieder suchen und über die Webseite kontaktieren.

www.smpa.org

Webseite der Schweizerischen MD-PhD Gesellschaft (SMPA). Die Mitgliedschaft ist für Studenten gratis und beträgt für Absolventen 30 Franken. Es finden sich viele nützliche Informationen und Links rund um das MD-PhD-Studium. Andere Mitglieder können ebenfalls gesucht und kontaktiert werden.
}

Dr. Ahn hatte zunächst seinen $\mathrm{PhD}$ auf dem Gebiet der molekularen Genetik erworben und danach beschlossen, Orthopäde zu werden. Er sei für einige Jahre in die Klinik abgetaucht, um gut in Chirurgie $\mathrm{zu}$ werden und habe erst danach vermehrt Forschung betrieben. Schon bald habe er eine eigene Forschungsgruppe mit einem Wissenschaftler zusammen gründen können. Diese Forschungsgruppe sei eine Partnerschaft, in der er vor allem Diskussionsbereitschaft, Organisation und Gelder mitbringe. Im Labor habe er hingegen wenig tägliche Arbeit. Für ihn müsse der Schwerpunkt während der Weiterbildung zuerst die Chirurgie und erst dann die Forschung sein. Ebenfalls sei es sehr empfehlenswert, sich während der Assistenzzeit mit klinischer Forschung und klinischen Studien vertraut zu machen.

\section{Austausch mit interessanten Persönlichkeiten}

Den Abschluss des Kongresses bildete ein Mittagessen mit Programmdirektoren, die klinische Stellen für MD-PhD-Absolventen ausschreiben. Viele der Stellen in Kombination mit Forschung wurden für Innere Medizin, Pathologie oder Pädiatrie angeboten. Eher seltener waren andere Fachrichtungen wie Neurologie oder Rheumatologie vertreten. Fast kein Universitätsspital hatte ein definiertes Programm für Chirurgie oder verwandte Disziplinen [3, 6]. Auf Nachfrage hiess es, man könne direkt Kontakt mit den Professoren in diesen Fachgebieten aufnehmen und um ein massgeschneidertes Programm verhandeln.

Nach dem Kongress gab es Gelegenheit, sich im Labor und in der Klinik für Transplantationsmedizin und -chirurgie an der Universität von Illinois in Chicago umzusehen. Die Besichtigung wurde von Jennifer Kwan geführt, MD-PhD und Assistenzärztin/Postdoc in der Nephrologie. Sie präsentierte das bestens ausgestattete Labor inklusive eigenem Sequenziergerät und Supercomputer. Im Anschluss folgte ein kurzer Besuch in der Sprechstunde für Nierentransplantierte, wo Dr. Kwan Patienten betreute. Sie erläuterte eines ihrer derzeit laufenden Projekte, bei dem sie durch Urin-, Blut- und Stuhlanalysen die Zusammenhänge zwischen mikrobiologischer Flora, Immunsystem und Transplantatfunktion zeigen will. Darüber hinaus betonte sie die einzigartige Möglichkeit, an ihrer Klinik diese Studien sowohl mit normalgewichtigen als auch mit stark übergewichtigen Patienten durchführen zu können.

Die ausgesprochen gute Vernetzung innerhalb des Spitals und zwischen den Spitälern war beeindruckend, insbesondere die Verfügbarkeit sämtlicher Krankenakten über das Internet auch ausserhalb des Spitalnetzwerks.

Im Gespräch machte Dr. Kwan auf drei Arten von Kliniken in den USA aufmerksam: erstens Student Hospitals, in denen Medizinstudenten arbeiten können. Dort werden auch unversicherte Patienten 
behandelt. Zweitens normale Spitäler, wo die Patienten für medizinische Leistungen versichert sein müssen. Drittens Veteranenspitäler, die vollständig von der US-Regierung getragen werden und Veteranen mit abgeleistetem Armeedienst nach den besten medizinischen Standards behandeln können.

\section{Karrieretipps}

Während des Laborbesuchs ergab sich die einmalige Möglichkeit, José Oberholzer zu treffen. Er leitete bis 2002 das Transplantationszentrum für Langerhanssche Inseln am Universitätsspital Genf. Danach wurde er an die Universität Illinois in Chicago berufen und leitet dort seit 2007 die gesamte Transplantationschirurgie. Dr. Oberholzer hat sich auf die Transplantation von Leber, Pankreas, Niere und Dünndarm mittels DaVinci-Operationsroboter spezialisiert. Diese Technik kommt vor allem stark übergewichtigen Patienten zugute. Dank des kleinen Zugangs und der kurzen Operationszeit erleiden sie viel weniger Komplikationen als bei einem offenen Eingriff. Wissenschaftlich beschäftigt sich Dr. Oberholzer ebenfalls mit Langerhansschen Inseln. Zum Beispiel werden in seinem Labor die Inseln verkapselt, um sie vor der Immunabwehr zu schützen. Fortschrittliche Kulturmethoden für die Langzeitzüchtung werden entwickelt. Tiermodelle werden eingesetzt, um die Transplantation im diabetischen Patienten zu simulieren. Oft treffe man auf die Ansicht, Chirurgie und Wissenschaft liessen sich nicht verbinden. Es sei aber durchaus möglich, spezialisierte chirurgische Eingriffe auf hohem Niveau durchzuführen und weiterhin interessante Forschung zu betreiben. Wichtig für diese Karriere seien gute Vorbilder, Durchhaltevermögen und die Fähigkeit, über den Tellerrand zu schauen. Bereits während seiner wissenschaftlichen Ausbildung habe er ausserdem darauf geachtet, sich um chirurgische Eingriffe am Menschen und an Tiermodellen zu bemühen. Dies und der anhaltende gute Kontakt zu seinen Mentoren hätten ihm geholfen, den Spagat zwischen Chirurgie und Wissenschaft zu bewältigen.

\section{Die Schweiz muss den internationalen Vergleich nicht scheuen}

Dieser Spagat ist die Herausforderung im Schnittpunkt zwischen Medizin und Forschung. Man läuft jederzeit Gefahr, von einer oder gar von beiden Seiten nicht mehr ernst genommen zu werden. Je nach Fachgebiet ist es mehr oder weniger schwierig, gute Vorbilder anzutreffen, die beide Seiten verbinden können.

Sowohl der Kongress als auch die anschliessenden Besuche sind empfehlenswerte Erfahrungen. Sie erlauben Einblick in ein sehr grosses und weit verzweigtes System mit vielen Ressourcen. Einerseits erscheinen das Forschungsprogramm und die Finanzierung als durchorganisiert, andererseits gibt es viel Freiraum und viele Chancen.

Amerika gilt nach wie vor als Land der unbegrenzten Möglichkeiten und man möchte nach den obigen Schilderungen meinen auch «zu Recht». Dennoch wird leicht vergessen, dass nur diejenigen wahrgenommen werden, die von diesen Möglichkeiten profitieren konnten. In der Schweiz bestehen nicht die gleichen Voraussetzungen, was Mittel, Freiraum und Chancen betrifft. Gleichzeitig ist der Spagat zwischen Medizin und Forschung ebenso schwierig. In Anbetracht der hohen Anzahl forschender Schweizer Ärzte dürfen wir aber auf keinen Fall den Vergleich scheuen. Unser Privileg ist auch eine starke politische und finanzielle Unterstützung, wie etwa durch den Schweizerischen Nationalfonds. Letztlich werden viele Ideen und Ansätze auf beiden Seiten des Atlantiks entwickelt und alle können von einem Austausch nur profitieren.

\section{Literatur}

1 Nguyen FT. The birth of the American Physician Scientists Association - the next generation of Young Turks. J Clin Invest. 2008;118(4):1237-40.

2 Banz Y, Feldmeyer L. SMPA - Swiss MD-PhD Association. Schweiz Ärztezeitung. 2010;91(11):441.

3 Brass LF, Akabas MH, Burnley LD, Engman DM, Wiley CA, Andersen OS. Are MD-PhD programs meeting their goals? An analysis of career choices made by graduates of $24 \mathrm{MD}-\mathrm{PhD}$ programs. Acad Med. 2010;85(4):692-701.

4 Jeffe DB, Andriole DA. A national cohort study of MD-PhD graduates of medical schools with and without funding from the National Institute of General Medical Sciences' Medical Scientist Training Program. Acad Med. 2011;86(8):953-61.

5 Kuehnle K, Winkler DT, Meier-Abt PJ. Swiss national MD-PhD-program: an outcome analysis. Swiss Med Wkly. 2009;19(37-38):540-6.

6 Wan DC, Wang KC, Longaker MT. Training the contemporary surgeon-scientist. Plast Reconstr Surg. 2012;129(4):1023-5. 